\title{
Genetics of atypical hemolytic uremic syndrome (aHUS)
}

Santiago Rodriguez de Cordoba, PhD. ${ }^{1,}$, Marta Subias Hidalgo, MSc ${ }^{1}$, Sheila Pinto, BTec $^{1}$ and Agustín Tortajada, $\mathrm{PhD}^{1}$

1) Centro de Investigaciones Biológicas, Consejo Superior de Investigaciones Científicas and Centro de Investigación Biomédica en Enfermedades Raras, Ramiro de Maeztu 9, 28040 Madrid, Spain.

Running Title: Genetics of atypical hemolytic uremic syndrome

*) Corresponding author: Prof. Santiago Rodríguez de Córdoba

Department of Cellular and Molecular Medicine

Centro de Investigaciones Biologicas

Ramiro de Maeztu 9,

Madrid 28040, Spain.

Tel: (+34) $917373112 \times 4432$

E-mail: srdecordoba@cib.csic.es 


\begin{abstract}
Hemolytic uremic syndrome (HUS) is a rare, life threatening disease characterized by thrombocytopenia, microangiopathic hemolytic anemia and acute renal failure. The atypical form of HUS (aHUS), representing 5-10\% of cases, lacks the association with infection by Shiga toxin producing E. coli strains that characterizes the commonest clinical presentation of HUS. In the majority of aHUS cases the disease results from the complement-mediated damage to the microvascular endothelium due to inherited defects in complement genes or autoantibodies against complement regulatory proteins. Incomplete penetrance of aHUS in carriers of mutations is common to all aHUS-associated complement genes and it is now established that the overall genetic predisposition to aHUS of an individual results from the combination of different inherited factors. Moreover, the patient's genotype influences the clinical evolution, the response to plasma therapies and the recurrence after transplantation. Here we describe the genetic component of aHUS, the lessons that we have learned from the functional characterization of the aHUS-associated mutations and the benefits of a comprehensive genetic analysis of the patients.
\end{abstract}

Keywords: Complement, aHUS, thrombotic microangiopathy. 
Since the early 1980's, atypical hemolytic uremic syndrome (aHUS) has been associated with complement abnormalities and in particular with activation of the alternative pathway. It was, however, the genome-wide linkage analysis performed in 1998 by Warwicker et al. which formally established the relationship between aHUS and the $R C A$ (regulator of complement activation) gene cluster at $1 \mathrm{q} 32$ encoding factor $\mathrm{H}$ and other complement regulatory proteins (1). These findings triggered a series of decisive studies that delineated the genetic predisposition to aHUS and revealed its pathogenic mechanisms, changing the earlier perception that aHUS was a pathology related to hypocomplementemia (lack of complement) to the realization that aHUS is a disorder involving tissue damage caused by dysregulated complement activation (2-8).

Most aHUS cases have a strong genetic component involving mutations (normally in heterozygosis) and polymorphisms in the genes encoding the complement regulatory proteins factor $\mathrm{H}(C F H)(1-3,5,6,9)$, membrane cofactor protein $(M C P)(10-12)$ and factor I $(C F I)(13,14)$, and in the genes encoding the complement components factor B $(C F B)$ (15) and $\mathrm{C} 3$ (C3) (16). In addition, genes in the coagulation pathway like thrombomodulin $(T H B D)$, which functions as a cofactor for thrombin to reduce blood coagulation and also regulates factor I-mediated $\mathrm{C} 3 \mathrm{~b}$ inactivation, and more recently plasminogen (PLG), a zymogen that is converted into plasmin and plays an important role in fibrinolysis, have also been implicated in aHUS $(17,18)$. Finally, it has recently been found that a subgroup of aHUS patients with a very early onset ( $<1$ year of age) carry mutations in the gene encoding diacylglycerol kinase- $\varepsilon(D G K E)$, a intracellular protein implicated in podocyte homeostasis and in modulating protein kinase $\mathrm{C}$ activity in endothelial cells and platelets $(19,20)$. A summary of the mutations in all these aHUSassociated genes is depicted in Figures 1, 2 and 3 and Table 1. 


\section{Complement regulators in aHUS}

Missense mutations in the $\mathrm{C}$-terminus of factor $\mathrm{H}$, a region that is critical to the capacity of the protein to bind cell surfaces and control local activation of complement, are prototypical of aHUS (21). CFH mutations are also the most prevalent genetic alteration, representing approximately $25 \%$ of the cases in all aHUS cohorts. Carriers of the Cterminal mutations express factor $\mathrm{H}$ molecules with a limited capacity to bind and protect cells from complement lysis $(4,5,7,8)$. Interestingly $\mathrm{C}$-terminal factor $\mathrm{H}$ mutations do not alter complement regulation in plasma and carriers of these mutations have normal levels of $\mathrm{C} 3$ in plasma. These findings are in agreement with the identification of aHUSassociated loss-of-function mutations in $M C P$ and $C F I$ for the reason that the $\mathrm{MCP}$ and factor I mutations also lead to an impaired protection of host cells from complement lysis without affecting significantly complement regulation in plasma (22). $\mathrm{CFH}$ mutations that lead to partial factor $\mathrm{H}$ deficiencies in aHUS patients also fit these ideas, as it is known for a long time that decreased levels of factor $\mathrm{H}$ affect primarily the complement regulation on surfaces (23).

The combination of both, an active complement system in plasma and a defective protection of cellular surfaces portrays aHUS as a situation of "autolesion" caused by the uncontrolled activation of complement on cell surfaces. By decreasing concentrations of factor $\mathrm{H}$ or factor I in plasma, or MCP on cell surfaces, aHUS-associated mutations predispose to disease. In a situation that triggers complement activation, carriers of aHUS predisposing factors cannot control deposition and amplification of $\mathrm{C} 3 \mathrm{~b}$ on the microvasculature cellular surfaces, which results in tissue damage and destruction. The surface dysregulation that characterizes aHUS is clearly distinct from the lack of complement regulation in plasma, leading to complete $\mathrm{C} 3$ consumption and severe 
hypocomplementemia that is typical, for example, of dense deposit disease (DDD) patients. In this respect, it is now widely accepted that the differential association of mutations and polymorphisms in factor $\mathrm{H}$ and other complement proteins with aHUS and other glomerulopathies, depending on whether they cause surface or fluid phase dysregulation, illustrate a clear distinction between the pathogenic mechanisms underlying these pathologies (24).

\section{aHUS-associated $\mathrm{CFH}-\mathrm{CFHRs}$ genomic rearrangements}

The factor H related proteins (FHRs) are relatively minor plasma components with concentrations in the range 5 to $50 \mathrm{mg} / \mathrm{L}$, presenting a high degree of similarity with factor H. Of particular interest is the almost complete sequence conservation in FHR1 of the Cterminal region of factor $\mathrm{H}$ (25). The genes CFHR3, CFHR1, CFHR4, CFHR2 and CFHR5 encoding these FHR proteins are located downstream (in that order) and closely linked to the $C F H$ gene within a region that shows significant genetic variability and it is characterized by the presence of large genomic duplications (ranging in size from 1.2 to 38 $\mathrm{kb})$ (5). These duplications make the region highly prone to genomic rearrangements through gene conversion and non-homologous recombination, which are readily identified by MLPA (Multiplex Ligation-dependent Probe Amplification) technologies, CNV (copy number variation) microarrays or western blots. Notably, several rearrangements have been identified in recent years associated with different pathologies involving complement dysregulation, including aHUS (24).

A very prevalent rearrangement in this region, a true common polymorphism in humans, is the deletion of the $C F H R 1$ and $C F H R 3$ genes resulting from a non-homologous recombination event between a duplicated region downstream of the $C F H$ and $C F H R 1$ genes. The deletion of the CFHRI and CFHR3 genes is included in a single extended 
CFH-CFHRs haplotype, $\mathrm{H} 4$, that associates with lower risk of age-related macular degeneration (AMD) (26) and IgA nephropathy (27) and increased risk of systemic lupus erythematosus (SLE) (28). Although it is currently unclear whether the deletion of the CFHR1 and CFHR3 genes by itself predisposes or protects from aHUS (29, 30), the frequency of homozygosity for the CFHR3-CFHR1 deletion is increased among aHUS patients as a consequence of the association between complete deficiency of the FHR1 protein and the generation of anti-factor $\mathrm{H}$ autoantibodies $(25,29)$. This is an intriguing association for which there is no clear explanation. Importantly, the anti-factor $\mathrm{H}$ autoantibodies recognize the C-terminus of factor $\mathrm{H}$ (31) and functionally mimic the prototypical factor $\mathrm{H}$ mutations that are frequently associated with the development of aHUS (32). Another relatively frequent rearrangement in the $C F H-C F H R$ region involves an unequal crossover between homologous regions in the 3' ends of CFHR3 and CFHR4 genes that specifically removes the $C F H R 1$ and $C F H R 4$ genes. This deletion is also found in aHUS patients in association with anti-factor $\mathrm{H}$ autoantibodies $(29,33)$.

Most interesting are the various genomic rearrangements between the 3' end exons of $\mathrm{CFH}$ and the homologous regions in CFHR1 or CFHR3, which have been associated with aHUS $(9,34)$. These rearrangements result in the generation of CFH::CFHR1 or $C F H:: C F H R 3$ hybrid genes that alter the $\mathrm{C}$-terminal region of factor $\mathrm{H}$, further illustrating the remarkable correlation between a dysfunctional $\mathrm{C}$-terminal region in factor $\mathrm{H}$ and aHUS. Similarly, the association of a CFHR1::CFH hybrid gene in which the C-terminal exons of FHR1 have been replaced by those in factor $\mathrm{H}$ (reversed $C F H:: C F H R 1$ hybrid gene) suggests that competing the binding of the $\mathrm{C}$-terminal region of factor $\mathrm{H}$ to substrates that are relevant in aHUS with a protein devoid of complement regulatory activity has the same consequences than the $\mathrm{C}$-terminal factor $\mathrm{H}$ mutations characteristic of aHUS (35). 


\section{Gain-of-function mutations in factor $B$ and $C 3$}

Factor B and C3 mutations are characteristic of a subgroup of aHUS patients showing persistent activation of the alternative pathway (AP) in plasma $(15,16,36)$. Importantly, while mutations in the complement regulators factor $\mathrm{H}, \mathrm{MCP}$ and factor I are loss-of-function mutations, mutations in factor B or C3 are gain-of-function mutations in the sense that they increase formation or stability of the $\mathrm{C} 3$ convertase or render it resistant to inactivation by the complement regulators $(15,16)$. These data unequivocally establish the critical role that dysregulation of the complement alternative pathway plays in the pathogenesis of aHUS and illustrate that complement dysregulation may result from either a defect in the regulatory proteins or an abnormally increased activity of the components of the $\mathrm{C} 3$ convertase of the alternative pathway.

From a pathogenic point of view it is intriguing that these factor B and C3 gain-offunction mutations, decreasing C3 plasma levels and causing different degrees of hypocomplementemia, are nevertheless associated with aHUS. One possible explanation is that increased complement activation caused by gain-of-function mutations, a situation that may be similar to that occurring during infection, coincides with an additional aHUS risk factor impairing surface protection. In support of this possibility, it has been noted that carriers of $C F B$ or $C 3$ gain-of-function mutations that develop aHUS are also carriers of a $M C P$ risk haplotype (see below) $(15,36)$. In the case of the mutations in $\mathrm{C} 3$ associated with aHUS, different experimental approaches have shown that these mutations alter the sensitivity of $\mathrm{C} 3 \mathrm{~b}$ to inactivation by factor $\mathrm{H}$ and $\mathrm{MCP}$ and/or change the susceptibility of the AP C3 convertase to accelerated decay by factor $\mathrm{H}$ and decay accelerating factor (DAF, CD55) (16, 36). This data again illustrate that aHUS associated mutations affect 
preferentially complement regulation on surfaces, which is in contrast with the fluid phase dysregulation caused by C3 mutations associated with other glomerulopathies $(36,37)$.

\section{Therapeutic implications}

The realization that aHUS is a disorder involving tissue damage caused by dysregulated alternative pathway complement activation provided strong support for the implementation of aHUS therapies based in the inhibition of the complement terminal pathway. Eculizumab, a blocking monoclonal antibody against human C5 that inhibits C5a release and terminal complement complex (TCC) formation in general, and membrane attack complex (MAC) generation on a cellular target, was first successfully used for treatment of paroxysmal nocturnal hemoglobinuria, a hemolytic and thrombotic disorder caused by deficiency of glycosylphosphatidylinisotol (GPI)-anchored complement regulators CD55 and CD59 on blood cells, which are therefore lysed by MAC formation. Eculizumab was successfully tested in aHUS patients, on a compassionate basis, to prevent relapses of the disease and recurrences after transplantation $(38,39)$. Later on, based on the excellent results obtained in phase II clinical trials during 2009-2010, eculizumab was approved by the US Food and Drugs Administration and the European Medicines Agency and has rapidly become the accepted therapy in patients with aHUS (40), both as a rescue therapy in acute episodes and as prophylaxis in labile patients and following renal transplant (41).

\section{Incomplete penetrance of aHUS in mutation carriers}

The penetrance of disease in carriers of mutations in any of the aHUS-associated genes is approximately $50 \%$, indicating that additional genetic and environmental factors contribute to disease development in these individuals. In this respect, it is now well 
documented that concurrence of different genetic risk factors, either combined mutations in more than one gene or the combination of mutations and risk polymorphisms, greatly influences predisposition to aHUS $(10,15,42,43)$.

The aHUS-associated polymorphisms are basically limited to three relatively frequent $C F H$ and $M C P$ haplotypes, which include both risk and protection factors (44). The common $\mathrm{CFH}$ haplotype $\mathrm{H} 2$ including the common polymorphism Val62Ile has been associated with lower risk to aHUS, AMD and DDD. Val62Ile lies within the N-terminal region that is essential for factor $\mathrm{H}$ regulatory activities. Consistent with the role of complement dysregulation in these pathologies it has been shown that the substitution of Val for Ile at position 62 increases the complement regulatory of factor $\mathrm{H}$ reducing activation of the complement alternative pathway. In fact, functional analyses have shown that the Val62Ile substitution in factor $\mathrm{H}$ increases the affinity for $\mathrm{C} 3 \mathrm{~b}$, competing more efficiently with factor $\mathrm{B}$ for $\mathrm{C} 3 \mathrm{~b}$ binding in the proconvertase formation and acquiring enhanced cofactor activity for the factor-I mediated proteolysis of C3b (45).

The CFH-H3 and MCPggaac haplotypes are the most relevant aHUS risk polymorphisms described thus far. Both haplotypes include SNPs located in the promoter region of $C F H$ and $M C P$ that have potential functional implications in the expression of factor $\mathrm{H}$ and $\mathrm{MCP}(3,10)$. Although additional studies are needed to fully characterize these haplotypes functionally, the association of $\mathrm{CFH}-\mathrm{H} 3$ and MCPggaac with aHUS is important because it may help to explain why some individuals are predisposed to aHUS in the absence of mutations in the known aHUS associated genes. It is also increasingly recognized that in carriers of mutations in these aHUS-associated genes, the $C F H$ and $M C P$ risk haplotypes may be needed for full manifestation of the disease $(10,15,36,42)$.

A recent collaborative study by the European Working Party on Complement Genetics in Renal Diseases in 795 patients with aHUS has identified that 3\% of these patients carry combined mutations, being combinations involving $M C P$ or $C F I$ mutations 
(25\%) more frequent than those with $C F H, C 3$ or $C F B$ mutations $(8 \%-10 \%)$. Furthermore, this large study also illustrated that the concomitant presence of $C F H$ and $M C P$ risk haplotypes significantly increased disease penetrance in combined mutation carriers, further suggesting that genotyping for the $C F H$ and $M C P$ risk haplotypes may help to predict risk of developing aHUS in affected carriers of mutations (42).

Apart from the $C F H$ and $M C P$ polymorphisms, other aHUS-associated polymorphisms in FHR proteins include the $\mathrm{FHR}-1 * \mathrm{~A} /{ }^{*} \mathrm{~B}$ variant; possession of the *B variant is risk for aHUS $(29,30)$. Polymorphisms in the CFHR5 gene have also been described and associated with risk of aHUS (46).

Taken together, genetic and functional analyses have established that aHUS involves complement alternative pathway dysregulation and develops as a consequence of defective protection of cellular surfaces from complement activation. Multiple hits, involving plasma and membrane-associated complement regulatory proteins as well as complement components, are likely required to cause dysregulation and significantly impair protection to host tissues. Environmental factors that activate complement likely modulate genetic predisposition and are also very important in aHUS. Infection, immunosuppressive drugs, cancer therapies, oral contraceptives, pregnancy and childbirth are important factors that trigger attacks of aHUS in some patients. In carriers of multiple strong aHUS genetic risk factors the contribution of the environment is probably minor. On the other hand, in those with a low genetic predisposition, strong environmental factors may still precipitate disease.

\section{Thrombomodulin and other coagulation genes}

The anticoagulant protein thrombomodulin, which functions as a cofactor for thrombin to reduce blood coagulation and also regulates factor I-mediated C3b inactivation, 
has been described associated with aHUS (18). Interestingly, functional analyses of these aHUS-associated THBD mutations supported a defect in the complement regulatory activities of this protein on cell surfaces, which is consistent with the complement dysregulation that characterize aHUS (18). However, it is currently unknown whether the anticoagulant activities of thrombomodulin are also disrupted by the aHUS-associated mutations and therefore may also be relevant in aHUS. In this regard, a very recent study has screened in 36 European-American sporadic aHUS patients, using targeted genomic enrichment and massive parallel sequencing, the coding sequences and splice sites in 85 genes, including all genes in both the complement and coagulation pathways (17). In addition to novel variants in various complement genes, the study found deleterious nonsynonymous rare variants in several coagulation genes. $P L G$, encoding plasminogen, a zymogen that is converted into plasmin and plays an important role in fibrinolysis, was the most frequently mutated gene (17). Although these data suggest that the coagulation pathway and PLG in particular also contribute to aHUS susceptibility, further studies are needed to confirm these associations and to determine the role of the coagulation pathway in aHUS.

\section{$D G K E$-aHUS}

Using exome sequencing Lemaire et al. (19) have recently identified recessive mutations in DGKE, encoding diacylglycerol kinase- $\varepsilon$, associated with aHUS in 13 patients from nine kindreds. Disease presentation in these patients occurred very early, typically before the age of 1 year, with multiple recurrences often progressing to end stage renal disease in the second decade of life. It has been indicated that $D G K E$ mutations may explain as much as $27 \%$ of cases presenting in the first year of life (19). An independent study also found recessive mutations in $D G K E$ in 9 patients from three consanguineous 
families with MPGN-like syndrome (20). Onset of disease in this second set of patients was significantly delayed and none of the patients in the study presented acute episodes suggesting aHUS. However, their histology showed features of glomerular microangiopathy, supporting that all patients carrying $D G K E$ mutations share a common pathogenic mechanism. Although the molecular basis is still unclear, one plausible explanation is that loss of $D G K E$ increases signaling through arachidonic acid containing diacylglycerol substrates, enhancing protein kinase $\mathrm{C}$ activation in endothelial cells, platelets and podocytes. As a consequence of this, prothrombotic factors like von Willebrand factor, plasminogen activator inhibitor-1, platelet-activating factor and tissue factor would be upregulated, leading to a thrombotic phenotype (19). In addition, loss of $D G K E$ may result in podocyte damage and impair slit diaphragm function, which could explain the heavy proteinuria observed in patients with $D G K E$ mutations (20).

Importantly, it was indicated that these individuals do not carry mutations in any of the known aHUS candidate complement genes and show no alterations of complement in plasma, suggesting that $D G K E$-associated aHUS represents an alternative mechanism leading to thrombotic microangiopathy (19). Although a role for complement dysregulation in the development of renal disease in carriers of $D G K E$ mutations seems $a$ priori excluded, we have recently identified three $D G K E$-associated early-onset aHUS patients carrying additional mutations in the $T H B D$ and $C 3$ genes. Further studies are therefore needed to determine whether complement dysregulation may influence the onset and severity of the disease phenotype in some carriers of $D G K E$ mutations.

\section{Genotype-phenotype correlations}

The clinical evolution of patients with aHUS, their response to plasma therapies and the disease recurrence after kidney transplantation are influenced by the type of 
mutation involved. In general, patients with $C F H, C F I, C F B$ and $C 3$ mutations have a worse prognosis during the episode of aHUS and following months, with rates of mortality and end stage renal disease rising to approximately $50 \%-70 \%$ and recurrences occurring in more than $50 \%$ of them. On the other hand, less than $20 \%$ of patients with $M C P$ mutations die or develop end stage renal disease, although the risk of recurrence is greater than $70 \%$ (47).

Historically, the success of kidney transplants in patients with end stage renal disease caused by aHUS has been limited by the high percentage of post-transplant recurrence of disease ( $\sim 50 \%$; graft loss rate: $80 \%-90 \%)$, although results vary based on the type of mutation present. $\mathrm{CFH}$ mutations are associated with a greater risk of recurrence or graft loss following renal transplantation (75\%-90\%), and high levels of risk are also associated with $C 3$ and $C F I$ mutations $(40 \%-80 \%)(42,47,48)$. Until now, very few transplants have been attempted in patients with $C F B$ mutations, but all cases that have been reported to date have involved recurrence of aHUS and graft lost.

Most complement factors involved in aHUS are plasma proteins primarily synthesised by the liver and thus patients with mutations of complement genes that code for these factors continue to be susceptible to aHUS after renal transplantation, since the dysfunctional factors continue to be produced. On the other hand, because MCP is a transmembrane protein primarily produced by the kidney, a kidney transplant corrects the deficit by producing unaltered MCP in the new kidney. Consequently, more than $80 \%$ of patients with $M C P$ mutations do not develop recurrent aHUS after transplantation, with a similar long-term survival rate to that of patients who receive transplants for other reasons. The risk of post-transplant recurrence in patients with $T H B D$ mutations or anti-factor $\mathrm{H}$ antibody mutations is not well understood, although in the case of factor $\mathrm{H}$ antibodies, it appears that recurrence is related to persistently high antibody titers $(31,47,48)$. 
Among patients with $C F H$ or $C F I$ mutations, the presence of mutations in other genes did not modify prognosis; in contrast, $50 \%$ of patients with combined $M C P$ mutation developed end stage renal disease within 3 years from onset compared with $19 \%$ of patients with isolated $M C P$ mutation. In general, a similar situation was observed for kidney transplant outcomes (42).

$D G K E$-aHUS illustrates a very particular subgroup of aHUS patients. As indicated above, $D G K E$-associated aHUS may represent an alternative mechanism involving intracellular signaling leading to thrombotic microangiopathy, independent of complement dysregulation. Consistent with this idea, two patients with $D G K E$ mutations who received plasma treatment or eculizumab, had aHUS recurrences. However, five other patients with $D G K E$ mutations underwent eculizumab treatment without evidence of disease recurrence (19). On the other side, a total of six aHUS patients with $D G K E$ mutations underwent renal transplantation without evidence of aHUS recurrence (19), but one transplanted patient with the MPGN-like form showed signs of disease recurrence in the transplanted kidney (20).

\section{Concluding remarks}

We have summarized our current understanding of the genetics of aHUS and reviewed how the functional analysis of different aHUS-associated genetic variants has helped to determine the molecular events that are critical in aHUS pathogenesis. It is now well established that mutations or polymorphisms in complement components and regulators are strongly associated with aHUS because they specifically impair the capacity to protect host cells from complement activation. It is also increasingly appreciated that it is the combination of mutations, or mutations and common polymorphisms in $\mathrm{CFH}$ and $M C P$, what determines the individual predisposition to aHUS and that this genetic make- 
up influences disease progression, responses to therapies and recurrences after transplantation. Based on these genotype-phenotype correlations it is also widely recognized that a comprehensive understanding of the genetic component predisposing to the pathology and its functional consequences at the protein level is critical to guide appropriate diagnostics and effective treatment in aHUS.

\section{Acknowledgements}

We thank Ángela Olimpia Ruiz and Daniel Sánchez Chinchilla for help in the preparation of the manuscript. The work of the authors described in this review was supported by the Spanish Ministerio de Economia y Competitividad (SAF2011-26583), the Comunidad de Madrid (S2010/BMD-2316), the Fundación Renal Iñigo Alvarez de Toledo and the Seventh Framework Programme European Union Project EURenOmics (European Consortium for High-Throughtput Research in Rare Kidney Diseases).

Abbreviations: aHUS, atypical Hemolytic Uremic Syndrome; AMD, Age-related Macular Degeneration; AP, alternative pathway; $\mathrm{CFB}$, complement factor B; $\mathrm{CFH}$, complement factor H; CNV, Copy Number Variation; DAF, decay accelerating factor; DDD, Dense Deposit Disease; DGKE, diacylglycerol kinase- $\varepsilon$; FHRs, Factor H Related proteins; GPI, glycosylphosphatidylinisotol; MLPA, Multiplex Ligation-dependent Probe Amplification; MAC, membrane attack complex; MCP, membrane cofactor protein; MPGN, Membranoproliferative Glomerulonephritis; PLG, plasminogen; RCA, regulator of complement activation; SLE, Systemic Lupus Erythematosus; TCC, terminal complement complex; THBD, thrombomodulin. 
Figure Legends.

\section{Figure 1, A-C. $C F H, M C P$ and $C F I$ mutations associated with aHUS}

Figure shows a schematic representation with the location of the mutations in the

protein domains of factor $\mathrm{H}, \mathrm{MCP}$ and factor I. Mutations that have been demonstrated to impair function or decrease expression levels are depicted in bold and underlined. Polymorphisms are shown in italics. References are in parenthesis. (*) Rodriguez de Cordoba et al. Unpublished Data.

\section{Figure 2. $C F B$ and $C 3$ mutations associated with aHUS}

Figure shows a schematic representation with the location of the mutations in the protein domains of factor B and C3. Mutations that have been demonstrated to impair function or decrease expression levels are depicted in bold and underlined. Polymorphisms are shown in italics. References are in parenthesis. (*) Rodriguez de Cordoba et al. Unpublished Data

\section{Figure 3. THBD and $D G K E$ mutations associated with aHUS}

Figure shows a schematic representation with the location of the mutations in the protein domains of Thrombomodulin and DGK- $\varepsilon$. Mutations that have been demonstrated to impair function or decrease expression levels are depicted in bold and underlined. References are in parenthesis. (*) Rodriguez de Cordoba et al. Unpublished Data 


\section{References}

1. Warwicker P, Goodship THJ, Donne RL, et al. Genetic studies into inherited and sporadic hemolytic uremic syndrome. Kidney Int 1998; 53(4): 836-44.

2. Caprioli J, Bettinaglio P, Zipfel PF, et al. The Molecular Basis of Familial Hemolytic Uremic Syndrome: Mutation Analysis of Factor H Gene Reveals a Hot Spot in Short Consensus Repeat 20. J Am Soc Nephrol 2001; 12(2): 297-307.

3. Caprioli J, Castelletti F, Bucchioni S, et al. Complement factor $\mathrm{H}$ mutations and gene polymorphisms in haemolytic uraemic syndrome: the C-257T, the A2089G and the G2881T polymorphisms are strongly associated with the disease. Hum Mol Genet 2003; 12(24): 3385-95.

4. Manuelian T, Hellwage J, Meri S, et al. Mutations in factor $\mathrm{H}$ reduce binding affinity to $\mathrm{C} 3 \mathrm{~b}$ and heparin and surface attachment to endothelial cells in hemolytic uremic syndrome. J Clin. Invest. 2003; 111(8): 1181-90.

5. Perez-Caballero D, Gonzalez-Rubio C, Gallardo ME, et al. Clustering of Missense Mutations in the C-Terminal Region of Factor $\mathrm{H}$ in Atypical Hemolytic Uremic Syndrome. Am. J. Hum. Genet. 2001; 68(2): 478-84.

6. Richards A, Buddles MR, Donne RL, et al. Factor H Mutations in Hemolytic Uremic Syndrome Cluster in Exons 18-20, a Domain Important for Host Cell Recognition. Am. J. Hum. Genet 2001; 68(2): 485-90.

7. Sanchez-Corral P, Gonzalez-Rubio C, Rodriguez de Cordoba S, et al. Functional analysis in serum from atypical Hemolytic Uremic Syndrome patients reveals impaired protection of host cells associated with mutations in factor H. Mol. Immunol. 2004; 41(1): 81-4.

8. Sanchez-Corral P, Perez-Caballero D, Huarte O, et al. Structural and Functional Characterization of Factor H Mutations Associated with Atypical Hemolytic Uremic Syndrome. Am. J. Hum. Genet 2002; 71(6): 1285-95.

9. Venables JP, Strain L, Routledge D, et al. Atypical Haemolytic Uraemic Syndrome Associated with a Hybrid Complement Gene. PLoS Med 2006; 3(10): e431.

10. Esparza-Gordillo J, Jorge EGd, Buil A, et al. Predisposition to atypical hemolytic uremic syndrome involves the concurrence of different susceptibility alleles in the 
regulators of complement activation gene cluster in 1q32. Hum Mol Genet 2005; 14(5): 703-12.

11. Noris M, Brioschi S, Caprioli J, et al. Familial haemolytic uraemic syndrome and an MCP mutation. Lancet 2003; 362(9395): 1542-7.

12. Richards A, Kemp EJ, Liszewski MK, et al. Mutations in human complement regulator, membrane cofactor protein (CD46), predispose to development of familial hemolytic uremic syndrome. Proc Natl Acad Sci U S A 2003; 100(22): 12966-71.

13. Fremeaux-Bacchi V, Dragon-Durey MA, Blouin J, et al. Complement factor I: a susceptibility gene for atypical haemolytic uraemic syndrome. J Med Genet 2004; 41(6): e84.

14. Kavanagh D, Kemp EJ, Mayland E, et al. Mutations in Complement Factor I Predispose to Development of Atypical Hemolytic Uremic Syndrome. J Am Soc Nephrol 2005; 16(7): 2150-5.

15. de Jorge EG, Harris CL, Esparza-Gordillo J, et al. Gain-of-function mutations in complement factor B are associated with atypical hemolytic uremic syndrome. Proc Natl Acad Sci U S A 2007; 104(1): 240-5.

16. Fremeaux-Bacchi V, Miller EC, Liszewski MK, et al. Mutations in complement C3 predispose to development of atypical hemolytic uremic syndrome. Blood 2008; 112(13): 4948-52.

17. Bu F, Maga T, Meyer NC, et al. Comprehensive Genetic Analysis of Complement and Coagulation Genes in Atypical Hemolytic Uremic Syndrome. J Am Soc Nephrol 2013: [Epub ahead of print].

18. Delvaeye M, Noris M, De Vriese A, et al. Thrombomodulin Mutations in Atypical Hemolytic Uremic Syndrome. N Engl J Med 2009; 361(4): 345-57.

19. Lemaire M, Fremeaux-Bacchi V, Schaefer F, et al. Recessive mutations in DGKE cause atypical hemolytic-uremic syndrome. Nat Genet 2013; 45(5): 531-6.

20. Ozaltin F, Li B, Rauhauser A, et al. DGKE Variants Cause a Glomerular Microangiopathy That Mimics Membranoproliferative GN. J Am Soc Nephrol 2013; 24(3): $377-84$. 
21. Rodriguez de Cordoba S, Esparza-Gordillo J, Goicoechea de Jorge E, et al. The human complement factor $\mathrm{H}$ : functional roles, genetic variations and disease associations. Mol. Immunol. 2004; 41(4): 355-67.

22. Atkinson JP, Liszewski MK, Richards A, et al. Hemolytic Uremic Syndrome: An Example of Insufficient Complement Regulation on Self-Tissue. Annals NY Acad. Sci. $2005 ; 1056(1): 144-52$.

23. Schreiber R, Pangburn M, Lesavre $P$, et al. Initiation of the alternative pathway of complement: recognition of activators by bound $\mathrm{C} 3 \mathrm{~b}$ and assembly of the entire pathway from six isolated proteins. Proc Natl Acad Sci U S A 1978; 75(8): 3948-52.

24. de Cordoba SR, Tortajada A, Harris CL, et al. Complement dysregulation and disease: From genes and proteins to diagnostics and drugs. Immunobiology 2012; 217(11): 1034-46.

25. Jozsi M, Zipfel PF. Factor $H$ family proteins and human diseases. Trends in Immunology 2008; 29(8): 380-7.

26. Hughes AE, Orr N, Esfandiary H, et al. A common CFH haplotype, with deletion of CFHR1 and CFHR3, is associated with lower risk of age-related macular degeneration. Nat Genet 2006; 38(10): 1173-7.

27. Gharavi AG, Kiryluk K, Choi M, et al. Genome-wide association study identifies susceptibility loci for IgA nephropathy. Nat Genet 2011; 43(4): 321-7.

28. Zhao J, Wu H, Khosravi M, et al. Association of Genetic Variants in Complement Factor $\mathrm{H}$ and Factor H-Related Genes with Systemic Lupus Erythematosus Susceptibility. PLoS Genet 2011; 7(5): e1002079.

29. Abarrategui-Garrido C, Martinez-Barricarte R, Lopez-Trascasa M, et al. Characterization of complement factor H-related (CFHR) proteins in plasma reveals novel genetic variations of CFHR1 associated with atypical hemolytic uremic syndrome. Blood 2009; 114(19): 4261-71.

30. Martinez-Barricarte R, Recalde S, Fernandez-Robredo P, et al. Relevance of Complement Factor H-Related 1 (CFHR1) Genotypes in Age-Related Macular Degeneration. Invest Ophthalmol Vis Sci 2012; 53(3): 1087-94.

31. Hofer J. Semin Thromb Haemost 2014. Current issue 
32. Jozsi M, Strobel S, Dahse H-M, et al. Anti factor H autoantibodies block C-terminal recognition function of factor $\mathrm{H}$ in hemolytic uremic syndrome. Blood 2007; 110(5): 1516-8.

33. Moore I, Strain L, Pappworth I, et al. Association of factor H autoantibodies with deletions of CFHR1, CFHR3, CFHR4, and with mutations in CFH, CFI, CD46, and C3 in patients with atypical hemolytic uremic syndrome. Blood 2010; 115(2): 379-87.

34. Francis NJ, McNicholas B, Awan A, et al. A novel hybrid CFH/CFHR3 gene generated by a microhomology-mediated deletion in familial atypical hemolytic uremic syndrome. Blood 2012; 119(2): 591-601.

35. Eyler S, Meyer N, Zhang Y, et al. A novel hybrid CFHR1/CFH gene causes atypical hemolytic uremic syndrome. Ped. Nephrol. 2013; 28(11): 2221-5.

36. Roumenina LT, Frimat M, Miller EC, et al. A prevalent C3 mutation in aHUS patients causes a direct C3 convertase gain of function. Blood 2012; 119(18): 4182-91.

37. Xiao X, Pickering MC, Smith RJH. C3 glomerulopathy: Dense deposit disease and C3 Glomerulonephritis. Semin Thromb. Haemost. 2014. Current issue

38. Mache CJ, Acham-Roschitz B, Fremeaux-Bacchi V, et al. Complement Inhibitor Eculizumab in Atypical Hemolytic Uremic Syndrome. Clin J Am Soc Nephrol 2009; 4(8): 1312-6.

39. Nurnberger J, Philipp T, Witzke O, et al. Eculizumab for Atypical Hemolytic-Uremic Syndrome. N Engl J Med 2009; 360(5): 542-4.

40. Riedl. Semin Thromb. Haemost. 2014. Current issue

41. Legendre CM, Licht C, Muus P, et al. Terminal Complement Inhibitor Eculizumab in Atypical Hemolytic-Uremic Syndrome. N Engl J Med 2013; 368(23): 2169-81.

42. Bresin E, Rurali E, Caprioli J, et al. Combined Complement Gene Mutations in Atypical Hemolytic Uremic Syndrome Influence Clinical Phenotype. J Am Soc Nephrol 2013; 24(3): 475-86.

43. Martinez-Barricarte R, Pianetti G, Gautard R, et al. The Complement Factor H R1210C Mutation Is Associated With Atypical Hemolytic Uremic Syndrome. J Am Soc Nephrol 2008; 19(3): 639-46. 
44. De Cordoba SR, De Jorge EG. Translational Mini-Review Series on Complement Factor H: Genetics and disease associations of human complement factor H. Clinical \& Experimental Immunology 2008; 151(1): 1-13.

45. Tortajada A, Montes T, Martinez-Barricarte R, et al. The disease-protective complement factor $\mathrm{H}$ allotypic variant Ile62 shows increased binding affinity for $\mathrm{C} 3 \mathrm{~b}$ and enhanced cofactor activity. Hum Mol Genet 2009; 18(18): 3452-61.

46. Monteferrante G, Brioschi S, Caprioli J, et al. Genetic analysis of the complement factor $\mathrm{H}$ related 5 gene in haemolytic uraemic syndrome. Mol. Immunol. 2007; 44(7): 1704-8.

47. Loirat C, Fremeaux-Bacchi V. Hemolytic uremic syndrome recurrence after renal transplantation. Pediatric Transplantation 2008; 12(6): 619-29.

48. Fremeaux-Bacchi V, Fakhouri F, Garnier A, et al. Genetics and Outcome of Atypical Hemolytic Uremic Syndrome: A Nationwide French Series Comparing Children and Adults. Clin J Am Soc Nephrol 2013; 8(4): 554-62.

49. Saunders RE, Goodship TH, Zipfel PF, et al. An interactive web database of factor Hassociated hemolytic uremic syndrome mutations: insights into the structural consequences of disease-associated mutations. Hum Mutat 2006; 27(1): 21-30.

50. Hocking HG, Herbert AP, Kavanagh D, et al. Structure of the N-terminal region of complement factor $\mathrm{H}$ and conformational implications of disease-linked sequence variations. J Biol Chem 2008; 283(14): 9475-87.

51. Saunders RE, Abarrategui-Garrido C, Fremeaux-Bacchi V, et al. The interactive Factor H-atypical hemolytic uremic syndrome mutation database and website: update and integration of membrane cofactor protein and Factor I mutations with structural models. Hum Mutat 2007; 28(3): 222-34.

52. Pechtl IC, Kavanagh D, McIntosh N, et al. Disease-associated N-terminal complement factor $\mathrm{H}$ mutations perturb cofactor and decay-accelerating activities. J Biol Chem 2011; 286(13): 11082-90.

53. Dragon-Durey MA, Fremeaux-Bacchi V, Loirat C, et al. Heterozygous and homozygous factor $\mathrm{h}$ deficiencies associated with hemolytic uremic syndrome or membranoproliferative glomerulonephritis: report and genetic analysis of 16 cases. $\mathrm{J}$ Am Soc Nephrol 2004; 15(3): 787-95. 
54. Falcao DA, Reis ES, Paixao-Cavalcante D, et al. Deficiency of the human complement regulatory protein factor $\mathrm{H}$ associated with low levels of component C9. Scand. J. Immunol. 2008; 68(4): 445-55.

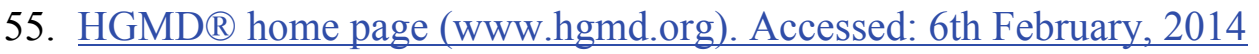

56. Sellier-Leclerc AL, Fremeaux-Bacchi V, Dragon-Durey MA, et al. Differential impact of complement mutations on clinical characteristics in atypical hemolytic uremic syndrome. J Am Soc Nephrol 2007; 18(8): 2392-400.

57. Sanchez-Corral P, Bellavia D, Amico L, et al. Molecular basis for factor H and FHL-1 deficiency in an Italian family. Immunogenetics 2000; 51(4-5): 366-9.

58. FH aHUS Mutation Database (C) (www.fh-hus.org). Accessed: 6th Fenruary, 2014.

59. Le Quintrec M, Lionet A, Kamar N, et al. Complement mutation-associated de novo thrombotic microangiopathy following kidney transplantation. Am J Transplant 2008; 8(8): 1694-701.

60. Vaziri-Sani F, Holmberg L, Sjoholm AG, et al. Phenotypic expression of factor H mutations in patients with atypical hemolytic uremic syndrome. Kidney Int 2006; 69(6): 981-8.

61. Neumann HP, Salzmann M, Bohnert-Iwan B, et al. Haemolytic uraemic syndrome and mutations of the factor $\mathrm{H}$ gene: a registry-based study of German speaking countries. $\mathrm{J}$ Med Genet 2003; 40(9): 676-81.

62. Hakobyan S, Tortajada A, Harris CL, et al. Variant-specific quantification of factor H in plasma identifies null alleles associated with atypical hemolytic uremic syndrome. Kidney Int 2010; 78(8): 782-8.

63. Stahl AL, Vaziri-Sani F, Heinen S, et al. Factor H dysfunction in patients with atypical hemolytic uremic syndrome contributes to complement deposition on platelets and their activation. Blood 2008; 111(11): 5307-15.

64. Caprioli J, Noris M, Brioschi S, et al. Genetics of HUS: the impact of MCP, CFH, and IF mutations on clinical presentation, response to treatment, and outcome. Blood 2006; 108(4): 1267-79.

65. Goodship TH. Factor H genotype-phenotype correlations: lessons from aHUS, MPGN II, and AMD. Kidney Int 2006; 70(1): 12-3. 
66. Cho HY, Lee BS, Moon KC, et al. Complete factor H deficiency-associated atypical hemolytic uremic syndrome in a neonate. Pediatr Nephrol 2007; 22(6): 874-80.

67. Ferreira VP, Herbert AP, Cortes C, et al. The binding of factor $\mathrm{H}$ to a complex of physiological polyanions and $\mathrm{C} 3 \mathrm{~b}$ on cells is impaired in atypical hemolytic uremic syndrome. J Immunol 2009; 182(11): 7009-18.

68. Herbert AP, Uhrin D, Lyon $\mathrm{M}$, et al. Disease-associated sequence variations congregate in a polyanion recognition patch on human factor $\mathrm{H}$ revealed in threedimensional structure. J Biol Chem 2006; 281(24): 16512-20.

69. Abarrategui-Garrido C, Melgosa M, Pena-Carrion A, et al. Mutations in proteins of the alternative pathway of complement and the pathogenesis of atypical hemolytic uremic syndrome. Am. J. Kidney Dis. 2008; 52(1): 171-80.

70. Jozsi M, Heinen S, Hartmann A, et al. Factor H and atypical hemolytic uremic syndrome: mutations in the C-terminus cause structural changes and defective recognition functions. J Am Soc Nephrol 2006; 17(1): 170-7.

71. Jokiranta TS, Jaakola VP, Lehtinen MJ, et al. Structure of complement factor H carboxyl-terminus reveals molecular basis of atypical haemolytic uremic syndrome. Embo j 2006; 25(8): 1784-94.

72. Filler G, Radhakrishnan S, Strain L, et al. Challenges in the management of infantile factor H associated hemolytic uremic syndrome. Pediatr Nephrol 2004; 19(8): 908-11.

73. Westra D, Volokhina E, van der Heijden E, et al. Genetic disorders in complement (regulating) genes in patients with atypical haemolytic uraemic syndrome (aHUS). Nephrol Dial Transplant 2010; 25(7): 2195-202.

74. Lehtinen MJ, Rops AL, Isenman DE, et al. Mutations of factor H impair regulation of surface-bound $\mathrm{C} 3 \mathrm{~b}$ by three mechanisms in atypical hemolytic uremic syndrome. $\mathrm{J}$ Biol Chem 2009; 284(23): 15650-8.

75. Provaznikova D, Rittich S, Malina M, et al. Manifestation of atypical hemolytic uremic syndrome caused by novel mutations in MCP. Pediatr Nephrol 2012; 27(1): 73-81.

76. Fan X, Yoshida Y, Honda S, et al. Analysis of genetic and predisposing factors in Japanese patients with atypical hemolytic uremic syndrome. Mol Immunol 2013; 54(2): 238-46. 
77. Noris M, Caprioli J, Bresin E, et al. Relative role of genetic complement abnormalities in sporadic and familial aHUS and their impact on clinical phenotype. Clin J Am Soc Nephrol 2010; 5(10): 1844-59.

78. Kwon T, Belot A, Ranchin B, et al. Varicella as a trigger of atypical haemolytic uraemic syndrome associated with complement dysfunction: two cases. Nephrol Dial Transplant 2009; 24(9): 2752-4.

79. Sullivan M, Erlic Z, Hoffmann MM, et al. Epidemiological approach to identifying genetic predispositions for atypical hemolytic uremic syndrome. Annals of human genetics 2010; 74(1): 17-26.

80. Fremeaux-Bacchi V, Moulton EA, Kavanagh D, et al. Genetic and functional analyses of membrane cofactor protein (CD46) mutations in atypical hemolytic uremic syndrome. J Am Soc Nephrol 2006; 17(7): 2017-25.

81. Cruzado JM, de Cordoba SR, Melilli E, et al. Successful renal transplantation in a patient with atypical hemolytic uremic syndrome carrying mutations in both factor I and MCP. Am J Transplant 2009; 9(6): 1477-83.

82. Servais A, Fremeaux-Bacchi V, Lequintrec M, et al. Primary glomerulonephritis with isolated C3 deposits: a new entity which shares common genetic risk factors with haemolytic uraemic syndrome. J Med Genet 2007; 44(3): 193-9.

83. Maga TK, Nishimura CJ, Weaver AE, et al. Mutations in alternative pathway complement proteins in American patients with atypical hemolytic uremic syndrome. Hum Mutat 2010; 31(6): E1445-60.

84. Bienaime F, Dragon-Durey MA, Regnier CH, et al. Mutations in components of complement influence the outcome of Factor I-associated atypical hemolytic uremic syndrome. Kidney Int 2010; 77(4): 339-49.

85. Nilsson SC, Kalchishkova N, Trouw LA, et al. Mutations in complement factor I as found in atypical hemolytic uremic syndrome lead to either altered secretion or altered function of factor I. Eur J Immunol 2010; 40(1): 172-85.

86. Kavanagh D, Richards A, Noris M, et al. Characterization of mutations in complement factor I (CFI) associated with hemolytic uremic syndrome. Mol Immunol 2008; 45(1): 95-105. 
87. Nilsson SC, Karpman D, Vaziri-Sani F, et al. A mutation in factor I that is associated with atypical hemolytic uremic syndrome does not affect the function of factor I in complement regulation. Mol Immunol 2007; 44(8): 1835-44.

88. Chan MR, Thomas CP, Torrealba JR, et al. Recurrent atypical hemolytic uremic syndrome associated with factor I mutation in a living related renal transplant recipient. Am. J. Kidney Dis. 2009; 53(2): 321-6.

89. Cayci FS, Cakar N, Hancer VS, et al. Eculizumab therapy in a child with hemolytic uremic syndrome and CFI mutation. Pediatr Nephrol 2012; 27(12): 2327-31.

90. Volokhina E, Westra D, Xue X, et al. Novel C3 mutation p.Lys65Gln in aHUS affects complement factor H binding. Pediatr Nephrol 2012; 27(9): 1519-24.

91. Sartz L, Olin AI, Kristoffersson AC, et al. A novel C3 mutation causing increased formation of the $\mathrm{C} 3$ convertase in familial atypical hemolytic uremic syndrome. $\mathrm{J}$ Immunol 2012; 188(4): 2030-7.

92. Marinozzi MC, Vergoz L, Rybkine T, et al. Complement factor B mutations in atypical hemolytic uremic syndrome - disease-relevant or benign? J Am Soc Nephrol In press.

93. Roumenina LT, Jablonski M, Hue C, et al. Hyperfunctional C3 convertase leads to complement deposition on endothelial cells and contributes to atypical hemolytic uremic syndrome. Blood 2009; 114(13): 2837-45.

94. Fremeaux-Bacchi V, Sanlaville D, Menouer S, et al. Unusual clinical severity of complement membrane cofactor protein-associated hemolytic-uremic syndrome and uniparental isodisomy. Am J Kidney Dis. 2007; 49(2): 323-9. 
W1183R (67), W1183L (8)

W1183* (68), T1184A (42)

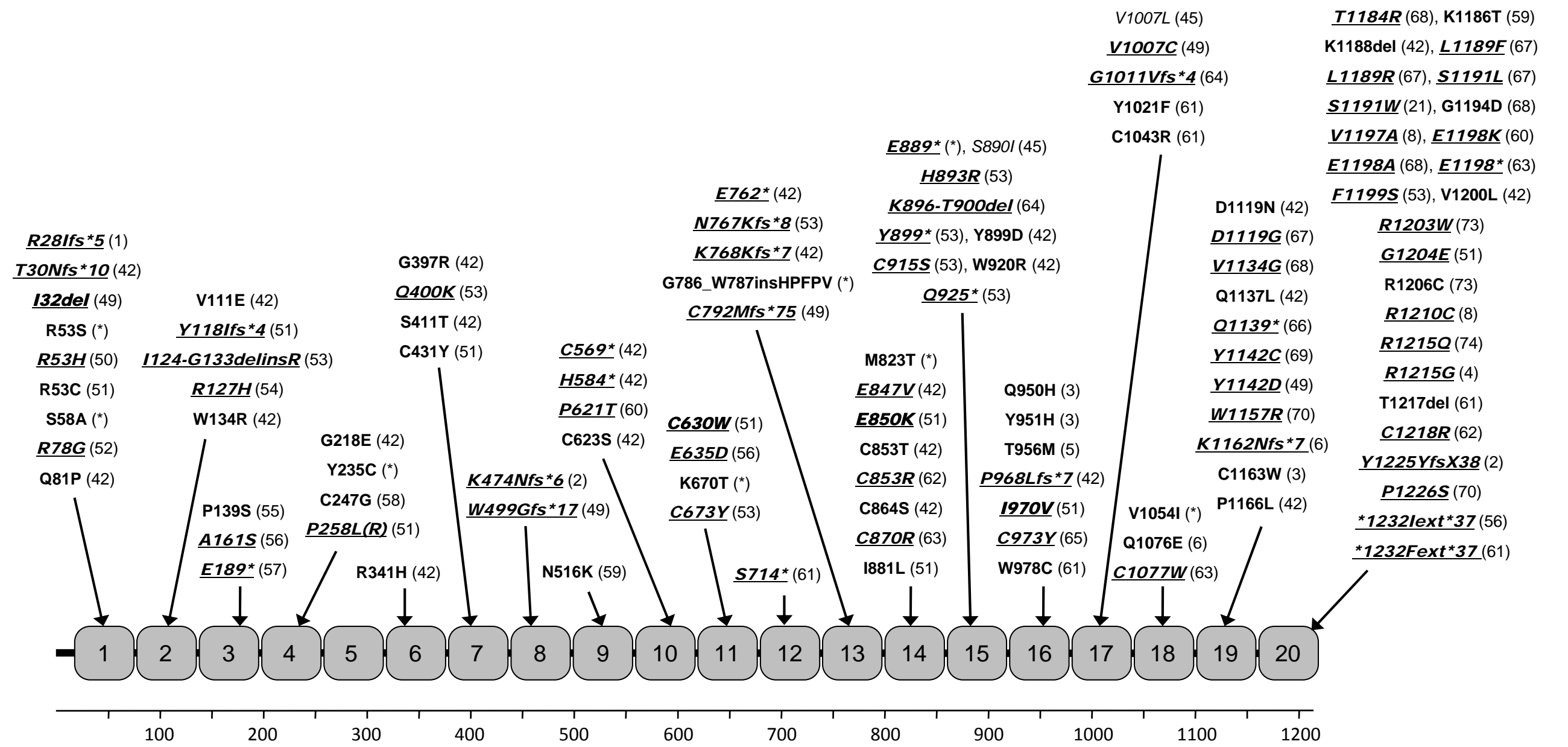




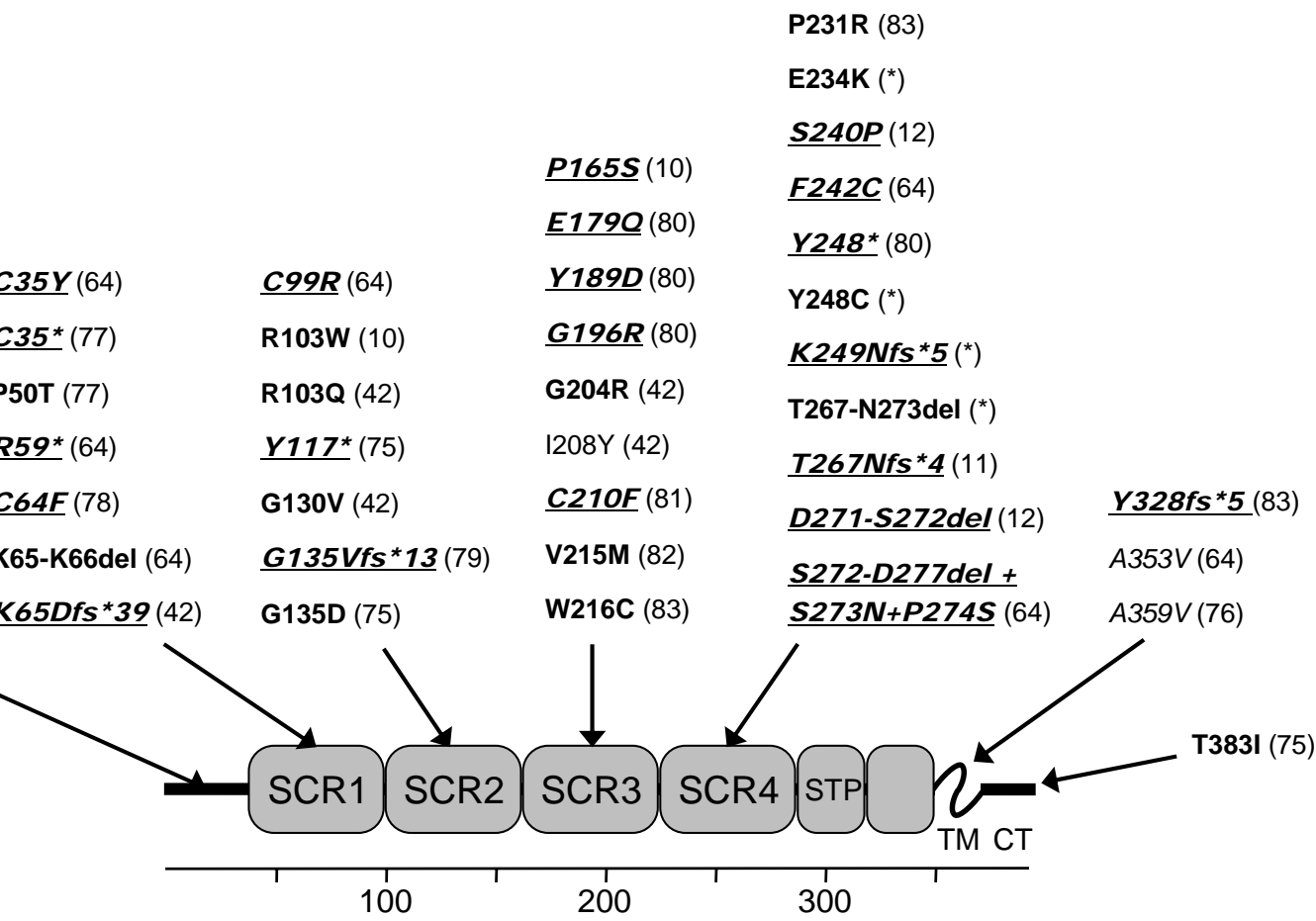

\section{CFI}

S298Lfs*10 (85)

E303K, E305* (58)

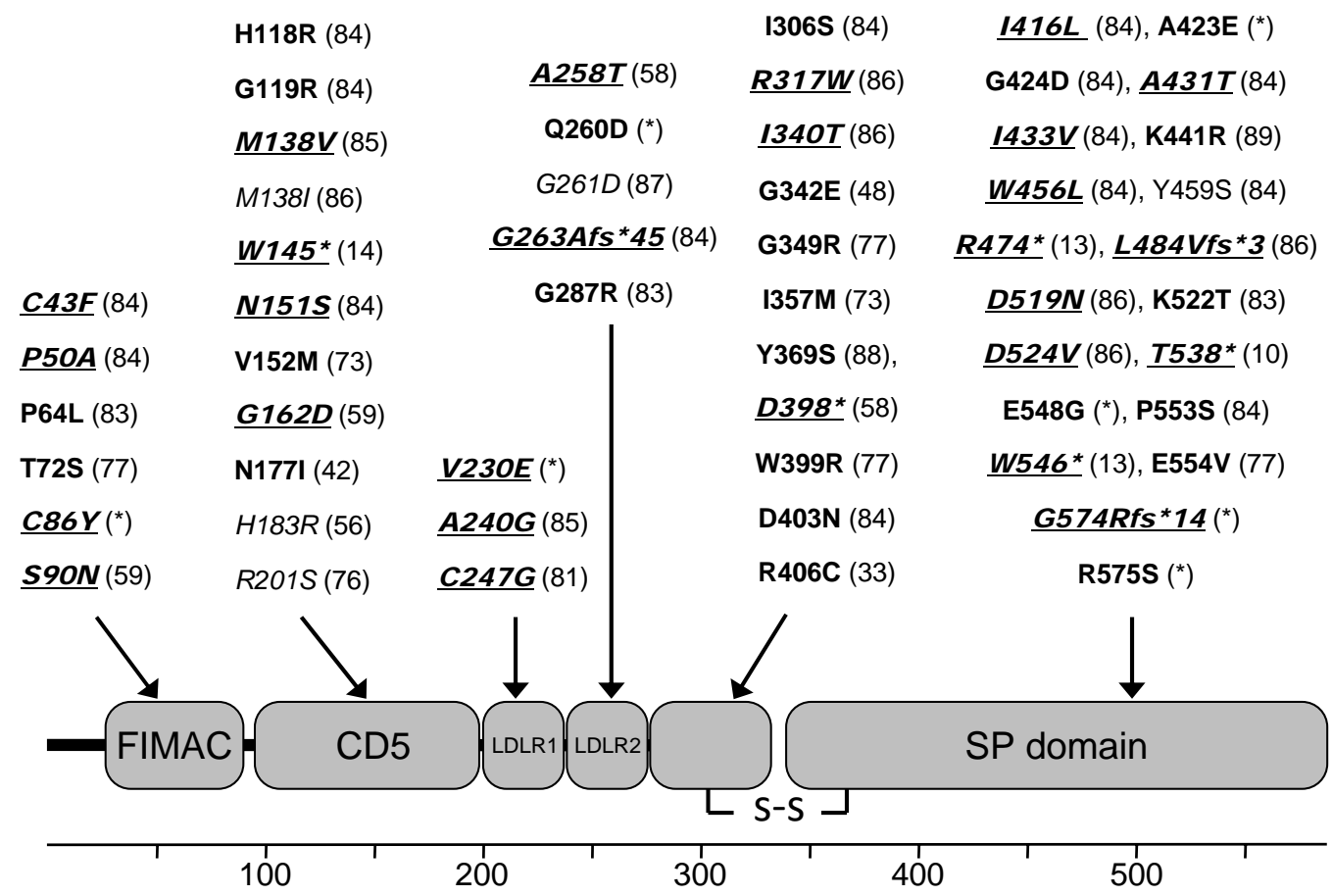




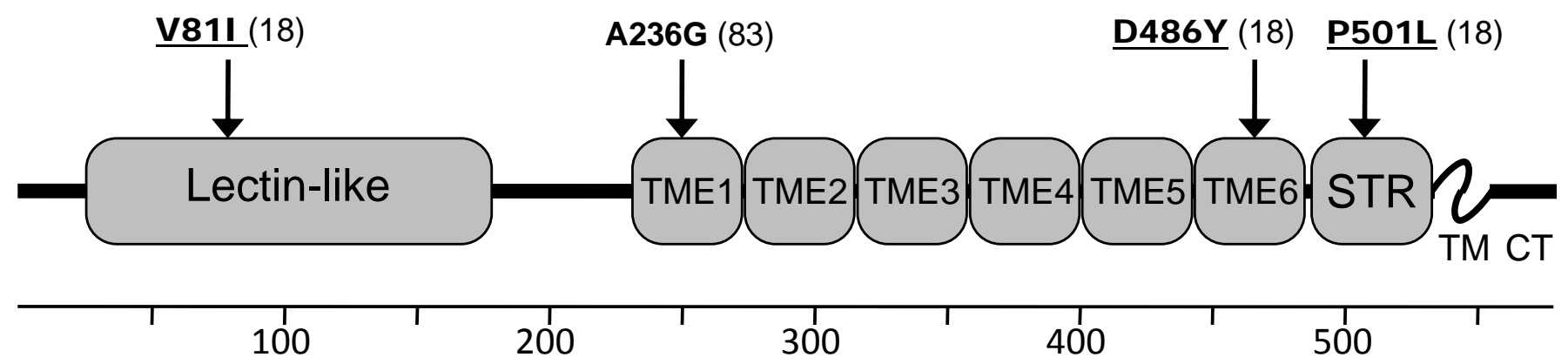

\section{DGKE}

$\underline{\mathrm{T} 204 \mathrm{Qfs} * 6}$ (20)

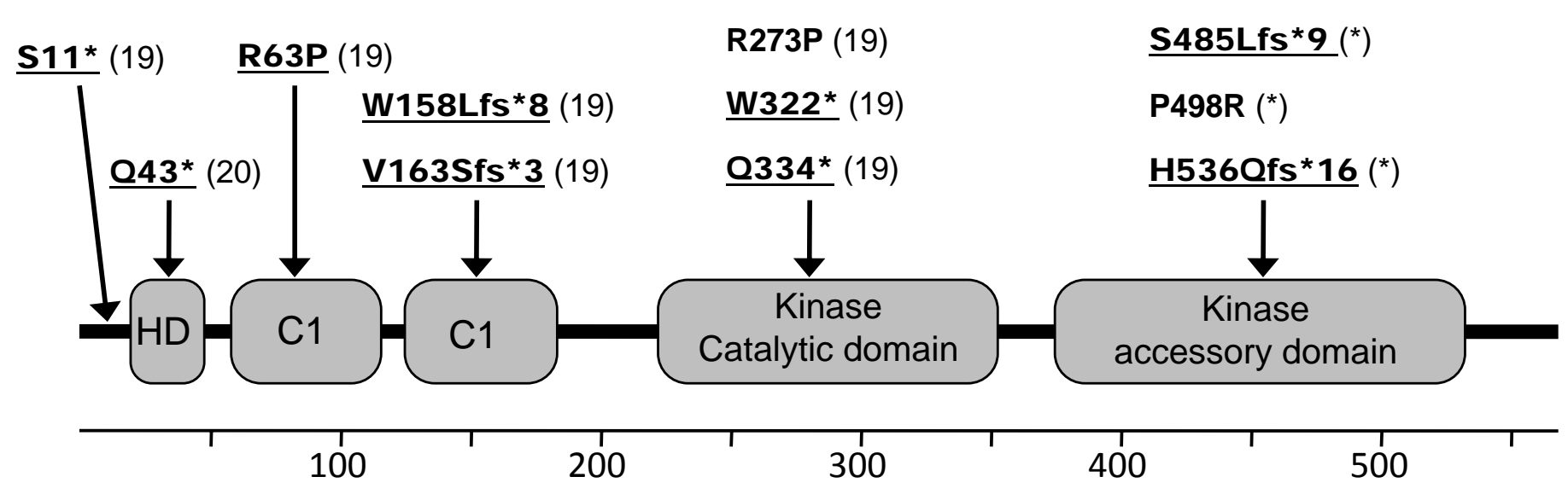

